\title{
(MIN, MAX)-EQUIVALENCE OF POSETS AND NONNEGATIVE TITS FORMS
}

\author{
V. M. Bondarenko ${ }^{1}$ and M. V. Stepochkina ${ }^{1}$ \\ UDC 512.64+512.56
}

\begin{abstract}
We study the relationship between the (min, max)-equivalence of posets and properties of their quadratic Tits form related to nonnegative definiteness. In particular, we prove that the Tits form of a poset $S$ is nonnegative definite if and only if the Tits form of any poset (min, max)-equivalent to $S$ is weakly nonnegative.
\end{abstract}

\section{Introduction}

Let $S$ be a finite poset that does not contain the element 0 . The quadratic form $q_{S}: \mathbb{Z} S \cup 0 \rightarrow \mathbb{Z}$ of this poset defined by the equality

$$
q_{S}(z)=z_{0}^{2}+\sum_{i \in S} z_{i}^{2}+\sum_{i<j, i, j \in S} z_{i} z_{j}-z_{0} \sum_{i \in S} z_{i}
$$

is called its Tits quadratic form. For the first time, this form was considered by Drozd [1], who showed that a poset $S$ has a finite (representation) type over a field $k$ if and only if its Tits form is weakly positive. It was shown in [2] that $S$ has the tame type if and only if the Tits form is weakly nonnegative.

Positive Tits forms ${ }^{2}$ and their applications in the theory of Tits representations were investigated in many works (see, e.g., [3-7]). The present paper is devoted to the study of posets with nonnegative Tits form.

We now recall the notion of the (min, max)-equivalence of posets [4].

For a minimal (respectively, maximal) element $a \in S$, we denote by $S_{a}^{\uparrow}$ (respectively, $S_{a}^{\downarrow}$ ) the poset $T=$ $T^{\prime} \cup\{a\}$, where $T^{\prime}=S \backslash\{a\}$ in the sense of posets (in this case, $T$ and $S$ are equal as ordinary sets) and the element $a$ is already maximal (respectively, minimal); furthermore, $a$ is comparable with $x$ in $T$ if and only if $a$ is incomparable with $x$ in $S$. We write $S_{x y}^{\uparrow \uparrow}$ instead of $\left(S_{x}^{\uparrow}\right)_{y}^{\uparrow}, S_{x y}^{\uparrow \downarrow}$ instead of $\left(S_{x}^{\uparrow}\right)_{y}^{\downarrow}$, etc.

A poset $T$ is called (min, max)-equivalent to a poset $S$ if $T$ is equal to a certain poset of the form

$$
\bar{S}=S_{x_{1} x_{2} \ldots x_{p}}^{\varepsilon_{1} \varepsilon_{2} \ldots \varepsilon_{p}}, \quad p \geq 0
$$

where $\varepsilon_{i} \in\{\uparrow, \downarrow\}$ and $x_{i}, i \in\{1, \ldots, p\}$, is a minimal (respectively, maximal) element of $S_{x_{1} x_{2} \ldots x_{i-1}}^{\varepsilon_{1} \varepsilon_{2} \ldots \varepsilon_{i-1}}$ if $\varepsilon_{i}=\uparrow\left(\right.$ respectively, $\left.\varepsilon_{i}=\downarrow\right)$; for $p=0$, we assume that $\bar{S}=S$. Moreover, the condition that the elements $x_{1}$, $x_{2}, \ldots, x_{p}$ are different is not necessary.

In the case where all $\varepsilon_{i}$ are equal to $\uparrow$ (respectively, $\downarrow$ ), we say that the poset $T$ is min-equivalent (respectively, max-equivalent) to the poset $S$. According to Corollary 2 and Proposition 11 in [6], the (min, max)-, min-, and max-equivalences are equivalence relations, and, furthermore, they are equivalent.

\footnotetext{
${ }^{1}$ Institute of Mathematics, Ukrainian National Academy of Sciences, Kiev, Ukraine.

${ }^{2}$ We use the term "positive form" instead of "positive-definite form" in connection with the conventional term "weakly positive form." The same is also true for nonnegative forms.
}

Translated from Ukrains'kyi Matematychnyi Zhurnal, Vol.60, No.9, pp. 1157-1167, September, 2008. Original article submitted February 5, 2008. 
Note that one can naturally extend the notion of (min, $\max$ )-equivalence to the notion of (min, $\max$ )-isomorphism by assuming that the posets $S$ and $S^{\prime}$ are (min, max)-isomorphic if there exists a poset $T$ that is (min, $\max$ )-equivalent to $S$ and isomorphic to $S^{\prime}$; the same is true for the min-equivalence and max-equivalence.

We now formulate the main results of the present paper.

Recall that a quadratic form $f(z)=f\left(z_{1}, \ldots, z_{m}\right): \mathbb{Z}^{m} \rightarrow \mathbb{Z}$ ( $\mathbb{Z}$ is the set of all integers) is called weakly nonnegative if it takes a nonnegative value on any vector with nonnegative coordinates. A form that takes nonnegative values on all vectors is called nonnegative (see Remark 1); in this case, we write $f(z) \geq 0$.

A poset $S$ is called NP-critical (respectively, WNP-critical) if the Tits form of any proper subset of it is nonnegative (respectively, weakly nonnegative), but the Tits form of $S$ itself does not possess this property.

The aim of the present paper is to prove the following theorems:

Theorem 1. For an arbitrary fixed poset $S$, the following assertions are true:

(1) if the Tits form of any poset min-equivalent to $S$ is weakly nonnegative, then the Tits form of $S$ itself is nonnegative;

(2) if the Tits form of $S$ is nonnegative, then the Tits form of any poset min-equivalent to $S$ is also nonnegative (and, a fortiori, it is weakly nonnegative).

Theorem 2. A poset $S$ is NP-critical if and only if it is min-equivalent to a certain WNP-2pt-critical poset.

In the conditions of Theorems 1 and 2, the min-equivalence can be replaced by the max-equivalence or by the (min, max)-equivalence (by virtue of their equivalence indicated above), as well as by the min-, max-, or (min, max)-isomorphism.

Note that WNP-critical posets (there are only six of them) are known (see Sec. 4). Theorem 2 gives an efficient method for the investigation of $N P$-critical sets.

Analogous results for positive and weakly positive Tits forms (along with many other results) were obtained by the authors in [6].

\section{Definitions and Notation for Posets}

Let $T=\left(T_{0}, \leq\right)$ be a poset. In what follows, a subset $X$ of the poset $T$ is always understood as a subset $X \subseteq T_{0}$ together with the induced relation of partial order, which will be denoted by the same symbol (in this case, for $x, y \in X$, the notation " $x \leq y$ in $T$ " is equivalent to the notation " $x \leq y$ in $X$ "); one-element subsets are identified with elements themselves. For simplicity, we write $x \in T$ instead of $x \in T_{0}, X \subset T$ instead of $X \subset T_{0}$, etc. (these natural simplifications have been used in Introduction).

A subset $X$ is called lower (respectively, upper) if $x \in X$ whenever $x<y$ (respectively, $x>y$ ) and $y \in X$, and it is called dense if $x \in X$ whenever $y<x<z$ and $y, z \in X$. It is obvious that lower and upper subsets are dense. Let $\overleftarrow{A}$ and $\vec{A}$, where $A$ is a subset of $T$, denote, respectively, the least lower subset and the least upper subset in $T$ that contain $A$. The subset $\overleftrightarrow{A}=\overleftarrow{A} \cap \vec{A}$, which is the least dense subset that contains $A$, is called the closure of the subset $A$ in $S$.

The notation $X<Y$ for subsets of $T$ means that $x<y$ for any $x \in X$ and $y \in Y$. Note that $Z<\varnothing$ and $\varnothing<Z$ for any subset $Z$. Further, the notation $x \gtrless y$ means that the elements $x$ and $y$ are incomparable. We set $T^{\ngtr}(a)=\{x \in T \mid x \gtrless a\}$. For an element $a \in T$, we denote by $\{a\}^{<}$(respectively, $\{a\}^{>}$) the subset of all $x \in T$ for which $x<a$ (respectively, $x>a$ ).

The maximum number of pairwise incomparable elements of a poset $T$ is called the width of this poset and is denoted by $w(T)$. 
We say that a poset $T$ is the sum of subsets $A$ and $B$ and write $T=A+B$ if $T=A \cup B$ and $A \cap B=\varnothing$. If $A<B$, then this sum is called ordinal, and if $x \ngtr y$ for any $x \in A$ and $y \in B$, then it is called direct. In the first case, we write $T=\{A<B\}$; in the second case, we write $T=A \coprod B$. These definitions can naturally be generalized to the case of an arbitrary number of subsets. A poset is called primitive if it is a direct sum of chains (linearly ordered sets).

\section{Properties of min-Equivalent Posets}

The min-equivalence of posets is denoted by $\cong_{\min }$ (the symbol $\cong$ denotes an isomorphism of posets). If $T_{2} \cong_{\min } T_{1}$, then, by definition, $T_{2}$ and $T_{1}$ are equal as ordinary sets. Therefore, every subset $X \subset T_{1}$ is also a subset in $T_{2}$, but not necessarily with the same partial order. If the order relation on $X$ has not been changed, then (to point out this fact) we often write $X^{\circ}$ instead of $X$ (for $X \subset T_{2}$ ).

Let $S$ be a poset. A finite sequence $\alpha=\left(x_{1}, x_{2}, \ldots, x_{p}\right)$ of elements $x_{i} \in S$ is called min-admissible if the expression $\bar{S}=S_{x_{1} x_{2} \ldots x_{p}}^{\uparrow \uparrow \uparrow}$ is meaningful (the case $p=0$ is not excluded). In this case, we also write $\bar{S}=S_{\alpha}^{\uparrow}$.

Let $\mathcal{P}(S)$ denote the set of all min-admissible sequences and let $\mathcal{P}_{1}(S)$ denote the set of all sequences of this type without repetitions. Denote the subset of $S$ that consists of all elements $x_{i}$ of a sequence $\alpha \in \mathcal{P}_{1}(S)$ by $[\alpha]_{S}$. Note that if $S$ and $T$ are min-equivalent, then there does not always exist $\alpha \in \mathcal{P}_{1}(S)$ such that $T=S_{\alpha}^{\uparrow}$ (see Sec. 6 in [6]).

According to Corollary 5 in [6], $\mathcal{P}_{1}(S)$ contains a sequence $\alpha$ such that $[\alpha]_{S}=X$ if and only if the subset $X$ is lower. According to Corollary 9 in [6], if $\alpha, \beta \in \mathcal{P}_{1}(S)$ and $[\alpha]_{S}=[\beta]_{S}$, then $S_{\alpha}^{\uparrow}=S_{\beta}^{\uparrow}$. Therefore, for the lower subset $X$, it is natural to define a poset $S_{X}^{\uparrow}$ by assuming that $S_{X}^{\uparrow}=S_{\alpha}^{\uparrow}$, where $\alpha \in \mathcal{P}_{1}(S)$ is an arbitrary sequence such that $[\alpha]_{S}=X$. It follows from Proposition 6 in [6] that, in $\bar{S}=S_{X}^{\uparrow}$, the subset $X$ is already upper and, hence, $Y=S \backslash X$ is lower (with the same partial orders); moreover, $y<x$ for $y \in Y$ and $x \in X$ (in $\bar{S}$ ) if and only if $y \gtrless x$ in $S$. In particular, if $S=X \coprod Y$ (respectively, $S=\{X<Y\}$ ), then $S_{X}^{\uparrow}=\{Y<X\}$ (respectively, $S_{X}^{\uparrow}=X \coprod Y$ ).

We now give several statements necessary for what follows. As above, $S$ is an arbitrary poset. Let $M_{-}(S)$ (respectively, $\left.M_{+}(S)\right)$ denote the set of all its minimal (respectively, maximal) elements.

Lemma 1 (lemma on cyclic permutation). Let $X=R \coprod\{M<N\}$ be a subset of a poset $S$. Then there exist $T_{1}, T_{2} \cong$ min $S$ in which $X=M^{\circ} \coprod\left\{N^{\circ}<R^{\circ}\right\}$ and $X=N^{\circ} \coprod\left\{R^{\circ}<M^{\circ}\right\}$, respectively.

Indeed, as $T_{1}$ and $T_{2}$, we can take the poset $T=S_{Y}^{\uparrow}$ for $Y=S \backslash \vec{N}$ and $Y=\overleftarrow{M}$, respectively.

Corollary 1. If $S$ contains subsets $A$ and $B$ such that $A<B$, then $A \cup B=A^{\circ} \amalg B^{\circ}$ in a certain $T \cong$ min $S$.

Indeed, one should set $M=A, N=B$, and $R=\varnothing$ in the conditions of the lemma.

Corollary 2. Suppose that $L=L_{1} \coprod \ldots \coprod L_{m}$ is a primitive subset of $S \quad\left(L_{1}, \ldots, L_{m}\right.$ are nonempty chains) and $c$ is an element of $S$ such that $c>L_{i}$ for any $i \neq m$ and $\{c\}^{<} \cap L_{m}=\varnothing$. Then there exists $T_{1} \cong_{\min } S$ that contains the primitive subset $L^{\prime}=L_{1}^{\circ} \ldots \amalg L_{m-1}^{\circ} \amalg L_{m}^{\prime}$, where $L_{m}^{\prime}$ is a chain of order $\left|L_{m}\right|+1$ that contains $L_{m}^{\circ}$.

Indeed, the case $w(L)<3$ is trivial. For $w(L) \geq 3$, one should use the lemma with $M=L_{1}+\ldots+L_{m-1}$, $N=\{c\}$, and $R=L_{m}$.

Lemma 2. Let $L$ be a dense subset of $S$. Then there exists $T \cong{ }_{\min } S$ in which $L$ is a lower subset with the same partial order. 
Indeed, as $T$, we can take $T=S_{P}^{\uparrow}$ for $P=\cup_{x \in M_{-}(L)}\{x\}<$.

In conclusion of this section, we give one statement in the general case (i.e., for sequences from $\mathcal{P}(S)$ ); this statement was proved in [6] (Lemma 26).

Proposition 1. Let $\alpha=\left(x_{1}, x_{2}, \ldots, x_{m}\right) \in \mathcal{P}(S)$, let $X$ be a subset of $S$, and let $\alpha_{X}$ be a subsequence of $\alpha$ that consists of all $x_{i} \in X$. Then $\alpha_{X} \in \mathcal{P}(X)$ and $X_{\alpha_{X}}^{\uparrow}$ is a subset of $S_{\alpha}^{\uparrow}$.

\section{Properties of a Quadratic Tits Form Related to Its Nonnegativity}

According to the main result of [4], quadratic Tits forms of min-equivalent posets are equivalent. In particular, this yields the following statement:

Proposition 2. Let $S$ and $T$ be min-equivalent posets. Then their Tits forms are simultaneously either nonnegative or not.

Recall that the ordinal sum $S=\left\{A_{1}<A_{2}<\ldots<A_{s}\right\}$ of antichains $A_{i}$ of lengths 1 and 2 (an antichain of length $m$ is a poset that consists of $m$ pairwise incomparable elements) is called a semichain. This is equivalent to the statement that $w(S)<3$ and $S$ does not contain subsets of width 2 of the form $\{a\} \coprod\{b<c\}$. The sets $A_{i}$ are called the links of a semichain. If all links are one-element, then $S$ is a chain.

Proposition 3. If the poset $S$ is a direct sum of two semichains, then its Tits form is nonnegative.

Proof. By virtue of Proposition 2 and the lemma on cyclic permutation for $X=S$ and $M=\varnothing$, it suffices to assume that $S$ is a semichain; moreover, we can obviously assume that all its links are two-element. Thus, let $S=\left\{A_{1}<A_{2}<\ldots<A_{s}\right\}$, where $A_{i}=\left\{i^{-}, i^{+}\right\}$. It is easy to see that

$$
2 q_{S}(z)=z_{0}^{2}+\sum_{i=1}^{s}\left(z_{i^{-}}-z_{i^{+}}\right)^{2}+\left(z_{0}-\sum_{j \in S} z_{j}\right)^{2}
$$

which implies that the form $q_{S}(z)$ is nonnegative. follows.

Finally, we give a statement on the nonnegativity of Tits forms for several specific posets necessary in what

Lemma 3. The quadratic Tits form is nonnegative for the following posets:

$$
\begin{gathered}
S_{1}=\{1 \prec 5,2 \prec 6,3 \prec 7,4 \prec 8,1 \prec 6,2 \prec 7,3 \prec 8,4 \prec 5\}, \\
S_{2}=\{2 \prec 5,3 \prec 6,4 \prec 7,2 \prec 6,3 \prec 7,4 \prec 5\}, \\
S_{3}=\{2 \prec 5,3 \prec 6,4 \prec 7,1 \prec 5,1 \prec 6,1 \prec 7\}, \\
S_{4}=\{2 \prec 4,5 \prec 6 \prec 7 \prec 8 \prec 9,3 \prec 4,3 \prec 6\}, \\
S_{5}=\{2 \prec 5 \prec 6,4 \prec 7 \prec 8,3 \prec 5,3 \prec 7\},
\end{gathered}
$$




$$
\begin{gathered}
S_{6}=\{1 \prec 4,2 \prec 5,6 \prec 7 \prec 8 \prec 9,2 \prec 4,3 \prec 5,3 \prec 7\}, \\
S_{7}=\{1 \prec 3,2 \prec 3,4 \prec 6,5 \prec 6,2 \prec 7,4 \prec 7,7 \prec 8\}, \\
S_{8}=\{1 \prec 3 \prec 4,6 \prec 7 \prec 8,2 \prec 3,2 \prec 9,5 \prec 7,5 \prec 9\}, \\
S_{9}=\{1 \prec 4 \prec 7,2 \prec 5 \prec 8,3 \prec 6 \prec 9,1 \prec 8,2 \prec 9,3 \prec 7\}, \\
S_{10}=\{1 \prec 2,3 \prec 4,5 \prec 6 \prec 7 \prec 8 \prec 9,3 \prec 7\}, \\
S_{11}=\{1 \prec 2,3 \prec 4 \prec 5,6 \prec 7 \prec 8 \prec 9,1 \prec 5,3 \prec 8\}, \\
S_{12}=\{1 \prec 2,3 \prec 4 \prec 5 \prec 6,7 \prec 8 \prec 9,1 \prec 5,3 \prec 9\}, \\
S_{13}=\{1 \prec 2 \prec 3,4 \prec 5 \prec 6,7 \prec 8 \prec 9,5 \prec 8\}, \\
S_{14}=\{2 \prec 3 \prec 4,5 \prec 6 \prec 7 \prec 8 \prec 9,2 \prec 8\} .
\end{gathered}
$$

It is assumed in the conditions of the lemma that each of the sets $S_{i}$ consists of the elements $1,2, \ldots, s$, where $s$ is the maximal number contained in its definition in explicit form.

The nonnegativity of the quadratic Tits form for the indicated posets was proved in [8] (see Lemma 4.3).

\section{WNP-Critical Posets}

Let $\langle p\rangle$ denote the chain $1<2<\ldots<p$ and let $\langle p, q, \ldots, r\rangle$ denote the direct sum of the chains $\langle p\rangle$, $\langle q\rangle, \ldots,\langle r\rangle$. We set $N=\{1 \prec 2,3 \prec 4,1 \prec 4\}$.

Proposition 4. A poset is WNP-critical if and only if it is isomorphic to one of the following posets: $\mathcal{N}_{1}=$ $\langle 1,1,1,1,1\rangle, \mathcal{N}_{2}=\langle 1,1,1,2\rangle, \mathcal{N}_{3}=\langle 2,2,3\rangle, \mathcal{N}_{4}=\langle 1,3,4\rangle, \mathcal{N}_{5}=\langle 1,2,6\rangle$, and $\mathcal{N}_{6}=N \coprod\langle 5\rangle$.

Proof. It follows from Theorem A in [2] and Proposition 3 in [1] that, first, any poset with not weakly nonnegative Tits form contains a certain $\mathcal{N}_{i}$ as a subset and, second, any proper subset of each $\mathcal{N}_{i}$ has a weakly nonnegative Tits form. In the proof of Theorem B in [2], it was shown that the Tits form of each $\mathcal{N}_{i}$ is not weakly nonnegative. These three facts imply that the proposition is true.

For the first time, the posets $\mathcal{N}_{1}-\mathcal{N}_{6}$ were introduced in Nazarova's work [9] devoted to the description of tame posets, and, therefore, we call them Nazarova critical sets. Their subsets $\mathcal{K}_{1}=\langle 1,1,1,1\rangle, \mathcal{K}_{2}=\langle 2,2,2\rangle$, $\mathcal{K}_{3}=\langle 1,3,3\rangle, \mathcal{K}_{4}=\langle 1,2,5\rangle$, and $\mathcal{K}_{5}=N \coprod\langle 4\rangle$ are called Kleiner critical sets; they were introduced in [10] and play the same role as the Nazarova sets, but in the description of posets of finite type.

In the case where $P$ is a given poset (say, $P=\mathcal{K}_{i}$ or $P=\mathcal{N}_{i}$ ), we say that a poset $T$ contains $P$ if $T$ contains $X$ isomorphic to $P$; if, in addition, $T=P$, then we say that $T$ is of the form $P$.

The statements presented below follow directly from definitions.

Lemma 4. The closure of a nondense subset of the form $\mathcal{K}_{i}$ contains a certain $\mathcal{N}_{j}$. 
Lemma 5. If a primitive poset $T$ contains a certain $\mathcal{K}_{i}$ as a proper subset, then it contains a certain $\mathcal{N}_{j}$.

Using the last lemma and Corollaries 1 and 2, we obtain the following statement:

Lemma 6. If a poset $S$ contains a certain primitive $K=\mathcal{K}_{i}$ and $x \in S$ is an element such that $K^{\prime}=$ $K \cap\{x\}^{<}$has the width $w \geq w(S)-1$ and is selected as a direct summand from $K$ (in particular, coincides with $K)$, then there exists $T \cong \min S$ that contains a certain $\mathcal{N}_{j}$.

One can obtain this statement by using Corollary 1 with $A=K$ and $B=x$ if $w\left(K^{\prime}\right)=w(S)$ (taking into account that $K^{\prime}=K$ in this case) and Corollary 2 with $L=K$ and $L_{m}=K \backslash K^{\prime}$ if $w\left(K^{\prime}\right)=w(S)-1$ and then applying Lemma 5.

We now prove the following statement:

Proposition 5. Any WNP-critical poset is NP-critical.

Proof. By definition, the Tits form of a WNP-critical set is not nonnegative. Further, using Proposition 4, one can easily show that any maximal subset $M$ of every $W N P$-critical set is either a subset (not necessarily proper) of a certain Kleiner critical set or a direct sum of two semichains the total number of two-element links of which does not exceed 1. In the first case, the Tits form of the set $M$ is nonnegative by virtue of Lemma 4.3 in [8]. In the second case, this statement is true by virtue of Proposition 3 (according to Proposition 21 in [6], the Tits form is positive in this case).

\section{Theorem on Posets without WNP-Critical Subsets}

Consider posets such that any posets min-equivalent to them do not contain Nazarova critical sets. Denote the collection of all these posets by $\mathcal{F}$.

The key role in the proof of Theorems 1 and 2 is played by the following statement:

Theorem 3. The Tits form of a set $S \in \mathcal{F}$ is nonnegative.

Note that it suffices to prove Theorem 3 for any fixed poset min-equivalent to $S$. We use this fact in what follows, choosing the most suitable poset in each individual case.

We now pass to the proof of Theorem 3. It is obvious that $w(S) \leq 4$ (otherwise $S \supset \mathcal{N}_{1}$ ). If any poset $T \cong$ min $S$ does not contain Kleiner critical sets, then, according to Proposition 24 in [6], the Tits form of the poset $S$ is positive. For this reason, we assume that $S$ contains at least one $\mathcal{K} \cong \mathcal{K}_{i}, 1 \leq i \leq 5$, and, furthermore, $S \neq \mathcal{K}$ because, by virtue of Lemma 3 , the posets $\mathcal{K}_{i}$ have nonnegative Tits forms.

First, we consider the case where $\mathcal{K} \cong \mathcal{K}_{1}$.

By virtue of Lemma 2 for $L=\mathcal{K}_{1}$, we can assume that $\mathcal{K}=M_{-}(S)$. Let $\mathcal{K}=\left\{a_{1}, a_{2}, a_{3}, a_{4}\right\}$. Denote the subset $\left\{a_{i}\right\}^{>} \cap\left\{a_{j}\right\}^{>}$by $L_{i j}$. In what follows, since $L_{j i}=L_{i j}$, considering these sets we always assume, for convenience, that $i<j$. Since $w(S)=4$ and $S \nsupseteq \mathcal{N}_{2}$, the union of all $\widehat{L}_{i j}=L_{i j} \cup\left\{a_{i}, a_{j}\right\}$ is equal to $S$. Furthermore, by virtue of Lemma 6, the subsets $L_{i j}$ and $L_{p q}$ do not intersect for $(i, j) \neq(p, q)$. Then each $L_{i j}$ is a semichain (possibly empty) because otherwise $\mathcal{K} \cup L_{i j}$ contains $\mathcal{N}_{1}$ or $\mathcal{N}_{2}$, depending on whether $L_{i j}$ contains the subset $X \cong\langle 1,1,1\rangle$ or $Y \cong\langle 1,2\rangle$.

If only one of the semichains $L_{i j}$ (of width 1 or 2 ) is nonempty or only two semichains $L_{i j}$ and $L_{p q}$ are nonempty for $\{i, j\} \cap\{p, q\}=\varnothing$, then $S$ is a direct sum of two semichains, and, by virtue of Proposition 3, we have $q_{S}(z) \geq 0$. This is also true for the case where there exists at least one $L_{i j}$ that is a semichain of width 2 ; indeed, in this case, each $L_{p q}$ is empty for $|\{i, j\} \cap\{p, q\}|=1$ because otherwise a subset that consists of two incomparable elements $a, b \in L_{i j}$, any element $c \in L_{p q}$, and elements of the subset $\mathcal{K} \backslash\left\{a_{i}, a_{j}\right\}$ (of order 2) is of the form $\mathcal{N}_{2}$. 
Thus, for $\mathcal{K} \cong \mathcal{K}_{1}$, it remains to consider the case where each $L_{i j}$ is a chain (possibly empty) and, furthermore, all $L_{i j}$ are pairwise disjoint and there exist $L_{p q}, L_{r s} \neq \varnothing$ such that $|\{p, q\} \cap\{r, s\}|=1$. We set $l_{i j}=\left|L_{i j}\right|$ and denote the number of nonempty $L_{i j}$ by $m=m(S)$.

Assume that, in this case, one of the following conditions is satisfied:

(a) $m=4$;

(b) $m=3$ and, for (pairwise different and nonempty) $L_{i j}, L_{p q}$, and $L_{r s}$, one has

$$
|\{i, j\} \cap\{p, q\}|=1, \quad|\{p, q\} \cap\{r, s\}|=1, \quad|\{i, j\} \cap\{r, s\}|=1, \quad|\{i, j\} \cap\{p, q\} \cap\{r, s\}|=0
$$

(c) $m=3$ and, for (pairwise different and nonempty) $L_{i j}, L_{p q}$, and $L_{r s}$, one has

$$
|\{i, j\} \cap\{p, q\} \cap\{r, s\}|=1 .
$$

Then (up to renumbering of minimal elements) one of the following cases takes place:

(1.1) $l_{12}=l_{23}=l_{34}=l_{14}=1$;

(1.2) $l_{12} \geq 1, l_{23} \geq 1, l_{34} \geq 1$, and $l_{14}>1$;

(2.1) $l_{12}=l_{23}=l_{13}=1$

(2.2) $l_{12} \geq 1, \quad l_{23} \geq 1$, and $l_{13}>1$

(3.1) $l_{12}=l_{13}=l_{14}=1$;

(3.2) $l_{12} \geq 1, l_{13} \geq 1$, and $l_{14}>1$.

Here, cases (1.1) and (1.2) correspond to condition (a), cases (2.1) and (2.2) correspond to condition (b), and cases (3.1) and (3.2) correspond to condition (c). Note that $l_{i j}$ not mentioned here are assumed to be zero. ${ }^{3}$

If none of conditions (a)-(c) is satisfied, then, up to renumbering of minimal elements, one has either $m=2$ and $L_{23}, L_{34} \neq \varnothing$ or $m=3$ and $L_{12}, L_{23}, L_{34} \neq \varnothing$. In these cases, we set $l=\left(l_{23}, l_{34}\right)$ and $l=\left(l_{12}, l_{23}, l_{34}\right)$, respectively, and assume (for special posets) that several coordinates of the vector $l$ can be defined not by a certain number but by inequalities of the form $>z$ and $\geq z$, where $z$ is a certain natural number, and by more usual inequalities of the form $z_{1} \leq s \leq z_{2}$. It is easy to see that, in this situation, one of the following cases takes place:

(4.1) $l=(1,1 \leq s \leq 4)$;

(4.2) $l=(1,>4)$;

(5.1) $l=(2,2)$;

(5.2) $l=(\geq 2,>2)$;

(6.1) $l=(1,1,1 \leq s \leq 3)$;

(6.2) $l=(1,1,>3)$;

${ }^{3}$ This assumption is also used in the investigation of the case $\mathcal{K} \cong \mathcal{K}_{i}$ for $i>1$. 
(7.1) $l=(1,2,1)$;

(7.2) $l=(1,>2,1)$;

(8.1) $l=(2,1,2)$;

(8.2) $l=(\geq 2,1,>2)$;

(9) $\quad l=(\geq 1,>1,>1)$.

Let us analyze cases (1.1)-(9).

In cases $(i .1), i=1,2, \ldots, 8$, the poset $S$ is contained, up to an isomorphism, in $S_{i}$ (see Lemma 3 ). In cases (1.2) and (2.2), $S$ contains $\mathcal{N}_{2}$; in cases (3.2), (7.2), and (9), it contains $\mathcal{N}_{3}$; in cases (5.2) and (8.2), it contains $\mathcal{N}_{4}$; in case (4.2), it contains $\mathcal{N}_{5}$; and in case (6.2), it contains $\mathcal{N}_{6}$. With regard for Lemma 3, this implies that if $S \in \mathcal{F}$, then its Tits form is nonnegative.

Now let $\mathcal{K} \cong \mathcal{K}_{i}$ for $i>1$. We assume that none of $T \cong$ min $S$ contains $\mathcal{K}_{1}$ because the case $\mathcal{K} \cong \mathcal{K}_{1}$ has already been considered. Then, according to Corollary 1 , the poset $T$ does not contain subsets of the form $Q_{13}=\left\{R_{1}<R_{3}\right\}, Q_{31}=\left\{R_{3}<R_{1}\right\}$, and $Q_{22}=\left\{R_{2}<R_{2}^{\prime}\right\}$, where $R_{1} \cong\langle 1\rangle$ is a set that consists of a single element $u_{0}, R_{2} \cong\langle 1,1\rangle$ (respectively, $R_{2}^{\prime} \cong\langle 1,1\rangle$ ) is a set that consists of two incomparable elements $u_{1}$ and $u_{2}$ (respectively, $u_{1}^{\prime}$ and $u_{2}^{\prime}$ ), and $R_{3} \cong\langle 1,1,1\rangle$ is a set that consists of three pairwise incomparable elements $v_{1}, v_{2}$, and $v_{3}$.

Further, according to Lemma 4, the subset $\mathcal{K}$ is dense. Then, by virtue of Lemma 2 for $L=\mathcal{K}_{i}$, we can assume that $\mathcal{K}$ is a lower subset of $S$. In particular, this yields $M_{-}(\mathcal{K})=M_{-}(S)$. We set $M_{-}(\mathcal{K})=\left\{a_{1}, a_{2}, a_{3}\right\}$ and $M_{+}(\mathcal{K})=\left\{b_{1}, b_{2}, b_{3}\right\}$ and assume that $a_{1} \leq b_{1}, a_{2}<b_{2}$, and $a_{3}<b_{3}$.

First, we consider the case $\mathcal{K} \cong \mathcal{K}_{i}$ for $i \neq 5$.

We set $B_{i j}=\left\{b_{i}\right\}^{>} \cap\left\{b_{j}\right\}^{>}$and $L_{i j}=\left\{a_{i}\right\}^{>} \cap\left\{b_{j}\right\}^{>}$(considering them only for $i \neq j$ ); we also set $C_{i}=\left\{b_{i}\right\}^{<} \cup b_{i}$. According to Lemma 5, $\mathcal{K}$ is a maximal primitive subset both in $S$ itself and in every $T \cong$ min $S$ in which $\mathcal{K} \cong \mathcal{K}_{i}$. Then, by virtue of Lemma 6 , we have $B_{i j}=\varnothing$, and, hence, $S \backslash \mathcal{K}$ is the union of all subsets $L_{i j}$ (otherwise $S$ contains $\mathcal{K}_{1}$ ), which are pairwise disjoint (otherwise $S \supset Q_{31}$ ). Furthermore, if $L_{i j}$ is nonempty, then $L_{i s}$ for $j \neq s$ and $L_{j i}$ are empty (otherwise $S \supset Q_{13}$ and $S \supset Q_{22}$, respectively). It follows from the relations $B_{i j}=\varnothing$ and $w(S)=3$ that $L_{i j}$ is a chain.

As in the case $\mathcal{K} \cong \mathcal{K}_{1}$, we denote the number of nonempty $L_{i j}$ by $m=m(S)$ and set $l_{i j}=\left|L_{i j}\right|$.

First, let $\mathcal{K} \cong \mathcal{K}_{2}$. If $m=3$, then, up to rearrangement of the numbers 1,2 , and 3 in subscripts, we get one of the following cases:

(10.1) $l_{12}=l_{23}=l_{31}=1$;

(10.2) $l_{12} \geq 1, l_{23} \geq 1$, and $l_{31}>1$.

If $m=1,2$, then one of the following cases (in which all $l_{i j}$ that are not mentioned are zero) takes place:

(11.1) $1 \leq l_{12} \leq 3$

(11.2) $l_{12}>3$;

(12.1) $l_{12}=1$ and $l_{23}=2$;

(12.2) $l_{12} \geq 1$ and $l_{23}>2$;

(13.1) $l_{12}=2$ and $l_{23}=1$;

(13.2) $l_{12}>2$ and $l_{23} \geq 1$. 
Now let us analyze cases (10.1)-(13.2).

In cases ( $i .1), i=10, \ldots, 13$, the poset $S$ is contained, up to an isomorphism, in $S_{i-1}$ (see Lemma 3). In cases (10.2), (11.2), (12.2), and (13.2), $S$ is contained in $\mathcal{N}_{3}, \mathcal{N}_{5}, \mathcal{N}_{6}$, and $\mathcal{N}_{4}$, respectively. With regard for Lemma 3, this implies that if $S \in \mathcal{F}$, then its Tits form is nonnegative.

Now let $\mathcal{K} \cong \mathcal{K}_{3}$. In this case, we can assume that $T \cong{ }_{\min } S$ does not contain $\mathcal{K}_{2}$ because the case $\mathcal{K} \cong \mathcal{K}_{2}$ has already been considered. According to the notation introduced above, $M_{-}(\mathcal{K})=\left\{a_{1}, a_{2}, a_{3}\right\}$ and $M_{+}(\mathcal{K})=\left\{b_{1}, b_{2}, b_{3}\right\}$, where $a_{1}=b_{1}, a_{2}<b_{2}$, and $a_{3}<b_{3}$. Let $c_{2}$ and $c_{3}$ denote the "missing" elements of the subset $\mathcal{K}: a_{2}<c_{2}<b_{2}$ and $a_{3}<c_{3}<b_{3}$.

Note that the set $K_{i j}=\left\{c_{i}\right\}^{>} \cap\left\{b_{j}\right\}^{>}$is empty if $i \neq j$ and $i, j \neq 1$ because otherwise, according to Corollary 2, for $L=L_{1} \coprod L_{2} \coprod L_{3}, L_{1}=\left\{a_{i}, c_{i}\right\}, L_{2}=\left\{a_{j}, c_{j}, b_{j}\right\}$, and $L_{3}=\left\{a_{1}\right\}$, a certain $T_{1} \cong_{\min } S$ contains $\mathcal{N}_{3}$. Further, $L_{i 1}, i=2,3$, coincides with $K_{i 1}$, otherwise $\mathcal{K} \cup\left(L_{i 1} \backslash K_{i 1}\right)$ contains $\mathcal{N}_{3}$. In this situation, if $L_{i 1} \neq \varnothing$, then $m=1$ because, in the case where $L_{i j} \neq \varnothing, j \neq 1$, the subset $\mathcal{K} \cup L_{i 1} \cup L_{i j}$ contains $Q_{13}$, and in the case where $L_{j i} \neq \varnothing, j \neq 1$, it contains $\mathcal{K}_{2}$.

Therefore, up to rearrangement of the numbers 2 and 3 in subscripts, one of the following cases takes place:

(14.1) $l_{21} \leq 2$

(14.2) $l_{21}>2$;

(15.1) $l_{23} \leq 2$;

(15.2) $l_{23}>2$.

In cases (14.1) and (15.1), the poset $S$, up to an isomorphism, is contained in $S_{13}$ and $S_{14}$, respectively (see Lemma 3). In cases (14.2) and (15.2), $S$ contains $\mathcal{N}_{4}$ and $\mathcal{N}_{5}$, respectively. Thus, for $S \in \mathcal{F}$, its Tits form is nonnegative.

We now show that, in the case $\mathcal{K} \cong \mathcal{K}_{4}$, there exists $T \cong{ }_{\text {min }} S$ that contains $\mathcal{K}_{2}$ or $\mathcal{K}_{3}$ (the corresponding cases have already been considered). According to the notation introduced above, we have $M_{-}(\mathcal{K})=\left\{a_{1}, a_{2}, a_{3}\right\}$ and $M_{+}(\mathcal{K})=\left\{b_{1}, b_{2}, b_{3}\right\}$, where $a_{1}=b_{1}, a_{2}<b_{2}$, and $a_{3}<b_{3}$. Let $c_{3}, d_{3}$, and $e_{3}$ denote the "missing" elements of the subset $\mathcal{K}: a_{3}<c_{3}<d_{3}<e_{3}<b_{3}$.

The subset $L_{23}$ is empty because otherwise, if $f$ denotes the maximal element of $L_{23}$, then $S_{P}^{\uparrow}$ with $P=$ $S \backslash f$ contains $\mathcal{N}_{6}$ (more exactly, $\mathcal{K} \cup f$ is of the form $\mathcal{N}_{6}$ ). If $L_{32} \neq \varnothing$ and $g \in L_{32}$, then $g>c_{3}$ because otherwise the subset $\left(\mathcal{K} \backslash a_{3}\right) \cup g$ is of the form $\mathcal{N}_{4}$; then, according to Corollary 2 for $L=L_{1} \amalg L_{2} \coprod L_{3}$, $L_{1}=\left\{a_{3}, c_{3}\right\}, L_{2}=C_{2}$, and $L_{3}=a_{1}$, there exists $T_{1} \cong$ min $S$ in which $\mathcal{K} \cup g$ is of the form $\mathcal{K}_{2}$. If $L_{31} \neq \varnothing$ and $h \in L_{31}$, then $h>d_{3}$ because otherwise $\left(\mathcal{K} \backslash\left\{a_{3}, c_{3}\right\} \cup h\right.$ is of the form $\mathcal{N}_{3}$. Then, according to Corollary 2 for $L=L_{1} \coprod L_{2} \coprod L_{3}, L_{1}=C_{1}, L_{2}=\left\{a_{3}, c_{3}, d_{3}\right\}$, and $L_{3}=C_{2}$, there exists $T_{1} \cong$ min $S$ in which $\mathcal{K} \cup h$ is of the form $\mathcal{K}_{3}$. Finally, if $L_{21} \neq \varnothing$ and $t \in L_{21}$, then $\mathcal{K} \cup t$ is of the form $\mathcal{N}_{6}$.

It remains to consider the case where $\mathcal{K} \cong \mathcal{K}_{5}$.

Let $U$ denote the subset of $\mathcal{K}$ that consists of the elements $a_{1}, b_{1}, a_{2}$, and $b_{2}$ and, furthermore, let $a_{1}<b_{2}$. Denote the "missing" elements of $\mathcal{K}$ by $c_{3}$ and $d_{3}$, assuming that $c_{3}<d_{3}$. Then $\mathcal{K}=U \amalg C_{3}$, where $C_{3}=\left\{a_{3}<c_{3}<d_{3}<b_{3}\right\}$. We set $C_{1}=\left\{a_{1}, b_{1}\right\}$ and $C_{2}=\left\{a_{2}, b_{2}\right\}$.

We need a statement that concretizes Corollary 2 (in the generality required for our purposes) and obviously follows from its proof.

Corollary 3. Suppose that $S, L=L_{1} \coprod \ldots \amalg L_{m}$, and $c$ are the same as in the conditions of Corollary 2, $m=3,\left|L_{1}\right|=i,\left|L_{2}\right|=j,\left|L_{3}\right|=\max (i, j)-1, i \leq j$, and $i+j=4$. Then there exists $T_{1} \cong \min S$ that contains $\mathcal{K}_{j}$. 
Let us show that the case $\mathcal{K} \cong \mathcal{K}_{5}$ reduces to the considered cases $\mathcal{K} \cong \mathcal{K}_{2}$ and $\mathcal{K} \cong \mathcal{K}_{3}$, namely, that there exists $T \cong$ min $S$ that contains $\mathcal{K}_{2}$ or $\mathcal{K}_{3}$.

We now assume that this is not true, i.e., that every poset $T$ min-equivalent to $S$ contains neither $\mathcal{K}_{2}$ nor $\mathcal{K}_{3}$, and show that this leads to a contradiction.

First, we show that $S$ is decomposable (with respect to the direct sum defined above). Assume that this is not true. Then there exists $x$ such that $\{x\}^{<} \cap U \neq \varnothing$ and $\{x\}^{<} \cap C_{3} \neq \varnothing$. Therefore, $x>a_{3}$. We set $R=\{x\}^{<} \cap U$. It is obvious that $b_{2} \notin R$ (otherwise $\mathcal{K} \cup x$ contains $Q_{31}$ ). For the same reason, $R$ cannot contain the elements $a_{1}$ and $a_{2}$ (respectively, $b_{1}$ and $a_{2}$ ) simultaneously. Furthermore, if $a_{1} \in R$, then $b_{1} \in R$, otherwise $\mathcal{K} \cup x$ contains $Q_{13}$. Thus, there are only two possibilities for $R$ : (a) $R=C_{1}$ and (b) $R=\left\{a_{2}\right\}$. Case (a) is impossible because, for $x \gtrless c_{3}$, the subset $\mathcal{K} \cup x$ contains $\mathcal{K}_{3}$, and for $x>c_{3}$, by virtue of Corollary 3 for $L_{1}=C_{1}, L_{2}=\left\{a_{3}, c_{3}\right\}, L_{3}=\left\{a_{2}\right\}$, and $c=x$, there exists $T_{1} \cong_{\min } S$ that contains $\mathcal{K}_{2}$. Case (b) is also impossible because, for $x \gtrless b_{3}$, the subset $M_{+}(\mathcal{K}) \cup x$ is of the form $\mathcal{K}_{1}$, and for $x>b_{3}$, by virtue of Corollary 3 for $L_{1}=a_{2}, L_{2}=C_{3} \backslash b_{3}, L_{3}=C_{1}$, and $c=x$, there exists $T_{1} \cong$ min $S$ that contains $\mathcal{K}_{3}$ (it is easy to see that the proof of Corollary 2 implies that there even exists $T_{1} \cong$ min $S$ that contains $\mathcal{N}_{4}$ ).

Thus, $S$ is decomposable into a direct sum of two proper subsets. It is clear that one of them contains $U$ and the other contains $C_{3}$. Therefore, there exists $x$ such that either $\{x\}^{<} \cap U=\varnothing$ and $\{x\}<\cap C_{3} \neq \varnothing$ or, vice versa, $\{x\}^{<} \cap U \neq \varnothing$ and $\{x\}^{<} \cap C_{3}=\varnothing$. In the first case, for $x \rtimes b_{3}$, the subset $M_{+}(\mathcal{K}) \cup x$ is of the form $\mathcal{K}_{1}$. For $x>b_{3}$, the subset $\mathcal{K} \cup x$ is of the form $\mathcal{N}_{6}$. Let us show that the second case is also impossible. We set $V=T^{\rtimes}(x) \cap U$. It is easy to see that $V$ is a subset of $U$ of width $w \leq 1$ (otherwise $\mathcal{K} \cup x$ contains $\mathcal{K}_{1}$ ); furthermore, $V$ is an upper subset because the subset $U \backslash V=\{x\}<\cap U$ is lower. In the case $w=1$, the subset $\mathcal{K} \cup x$ also contains $Q_{22}$ if $V=\left\{b_{2}\right\}$ and $\mathcal{N}_{4}$ if $V=\left\{b_{1}\right\}$ or $V=C_{2}$. If $V$ is empty, then, according to the lemma on cyclic rearrangement (for $M=U, N=x$, and $R=C_{3}$ ), there exists $T_{1} \cong$ min $S$ in which $\mathcal{K} \cup x$ is of the form $\mathcal{N}_{6}$.

Thus, we arrive at a contradiction. Therefore, there exists $T \cong \cong_{\min } S$ that contains $\mathcal{K}_{2}$ or $\mathcal{K}_{3}$.

Theorem 3 is proved.

\section{Proof of Theorems 1 and 2}

We can now easily prove Theorems 1 and 2 .

First, we prove Theorem 2. If the poset $S$ is min-equivalent to the $W N P$-critical set $\mathcal{N}$, then, by virtue of Propositions 2 and 5, the Tits form $q_{S}(z)$ is not nonnegative. It is easy to see that Proposition 1, with regard for Propositions 2 and 5, implies that every proper subset $R \subset S$ has a nonnegative Tits form. Indeed, otherwise $\mathcal{N}$ has a proper subset $Q \cong$ min $R$ whose Tits form is not nonnegative, which contradicts the fact that the set $\mathcal{N}$ is $N P$-critical. Thus, $S$ is $N P$-critical.

Conversely, if $S$ is $N P$-critical, then, according to Theorem 3, it is min-equivalent to a certain poset $S^{\prime}$ that contains a $W N P$-critical set $N \cong \mathcal{N}_{i}$. In this case, again by virtue of Propositions 1 and 2, we have $S^{\prime}=N$, and, hence, $S$ is min-equivalent to $N$.

We now pass to the proof of Theorem 1. Assertion (2) of the theorem follows directly from Proposition 2. If $S$ satisfies the condition of assertion (1), then any poset min-equivalent to $S$ does not contain $W N P$-critical subsets (by virtue of the definition of the latter). Therefore, according to Theorem 3, $S$ has a nonnegative Tits form.

\section{REFERENCES}

1. Yu. A. Drozd, "Coxeter transformations and representations of posets," Funkts. Anal. Prilozhen., 8, 34-42 (1974).

2. A. G. Zavadskii and L. A. Nazarova, "Posets of the tame type," in: Matrix Problems [in Russian], Institute of Mathematics, Ukrainian Academy of Sciences, Kiev (1977), pp. 122-143.

3. V. M. Bondarenko and A. M. Polishchuk, "On a criterion for the positive definiteness of one class of infinite quadratic forms," Nelin. Kolyvannya, 6, No. 1, 3-14 (2003). 
4. V. M. Bondarenko, "On (min, max)-equivalence of posets and applications to the Tits forms," Visn. Kyiv Univ., Ser. Fiz. Mat., No. 1, 24-25 (2005).

5. V. M. Bondarenko and M. V. Styopochkina, "On posets of width two with positive Tits form," Algebra Discr. Math., No. 2, 11-22 (2005).

6. V. M. Bondarenko and M. V. Stepochkina, “(Min, max)-equivalence of posets and quadratic Tits form," in: Problems of Analysis and Algebra, Vol. 2, Issue 3, Institute of Mathematics, Ukrainian National Academy of Sciences, Kiev (2005), pp. 18-58.

7. V. M. Bondarenko and M. V. Styopochkina, "On finite posets of infinite type and their Tits forms," Algebra Discr. Math., No. 2, 17-21 (2006).

8. V. M. Bondarenko, A. G. Zavadskii, and L. A. Nazarova, "On representations of tame posets," in: Representations and Quadratic Forms [in Russian], Institute of Mathematics, Ukrainian Academy of Sciences, Kiev (1979), pp. 75-106.

9. L. A. Nazarova, "Posets of infinite type," Izv. Akad. Nauk SSSR, Ser. Mat., 39, No. 5, 963-991 (1975).

10. M. M. Kleiner, "Posets of finite type," Zap. Nauch. Sem. LOMI, 28, 32-41 (1972). 